\title{
Brief report: Repetitive behaviour profiles in Williams syndrome: Cross syndrome comparisons with Prader-Willi and Down syndromes
}

Royston, R., Oliver, C., Moss, J., Adams, D., Berg, K., Burbidge, C., Howlin, P., Nelson, L., Stinton, C. \& Waite, J.

Cerebra Centre for Neurodevelopmental Disorders,

School of Psychology,

University of Birmingham

Please use this reference when citing this work: Royston, R., Oliver, C., Moss, J., Adams, D., Berg, K., Burbidge, C., Howlin, P., Nelson, L., Stinton, C. \& Waite, J. Brief Report: Repetitive behaviour profiles in Williams syndrome: Cross syndrome comparisons with Prader-Willi and Down syndromes. Journal of Autism and Developmental Disorders, 1-6, DOI 10.1007/s10803-017-3319-6

\section{The Cerebra Centre for Neurodevelopmental Disorders,}

School of Psychology, University of Birmingham, Edgbaston, Birmingham, B15 2TT

Website: www.cndd.Bham.ac.uk E-mail: cndd-enquiries@contacts.bham.ac.uk 


\begin{abstract}
This study describes the profile of repetitive behaviour in individuals with Williams syndrome, utilising cross-syndrome comparisons with people with Prader-Willi and Down syndromes. The Repetitive Behaviour Questionnaire was administered to caregivers of adults with Williams ( $n=96)$, Prader-Willi $(n=103)$ and Down $(n=78)$ syndromes. There were few group differences, although participants with Williams syndrome were more likely to show body stereotypies. Individuals with Williams syndrome also showed more hoarding and less tidying behaviours than those with Down syndrome. IQ and adaptive ability were negatively associated with repetitive questioning in people with Williams syndrome. The profile of repetitive behaviour amongst individuals with Williams syndrome was similar to the comparison syndromes. The cognitive mechanisms underlying these behaviours in genetic syndromes warrant further investigation.
\end{abstract}




\section{Brief Report: Repetitive behaviour profiles in Williams syndrome: Cross syndrome comparisons with Prader-Willi and Down syndromes.}

Williams syndrome (WS) is caused by a microdeletion on chromosome 7q11.23 and affects approximately 1 in 7500 individuals (Pober 2010; Stromme et al. 2002). It is associated with behavioural, cognitive, physical and emotional characteristics and mild to moderate intellectual disability (ID; Martens et al., 2008; Pober 2010).

Repetitive behaviour is an umbrella term for behaviours that are characterised by being contextually incongruous, and are repeated frequently, with little variability or appropriateness (Turner 1997). Whilst repetitive behaviours have been extensively researched in autism spectrum disorder (ASD), these behaviours are characteristic of many other neurodevelopmental disorders including Williams, Fragile-X, Prader-Willi, Lowe, Cornelia de Lange and Rubinstein-Taybi syndromes (Janes et al., 2014; Moss et al. 2009; Waite et al. 2015).

Moss et al. (2009) demonstrated the utility of examining repetitive behaviour profiles at a finegrained level amongst people with genetic syndromes, identifying syndrome specific differences between individuals with Angelman, Cornelia de Lange, Cri-du-Chat, Fragile X, Prader-Willi, Lowe and Smith-Magenis syndromes. However, although repetitive behaviour has been reported in 86\% of individuals with WS (Davies et al. 1998; Janes et al. 2014), there is little research delineating specific repetitive behaviour profiles in this condition. The literature is further limited by a lack of adult-focused research, small samples, and the use of assessments examining repetitive behaviours at only a broad level (Janes et al. 2014; Lincoln et al. 2007). More detailed knowledge about the profile of repetitive behaviour in people with WS is important, given evidence that repetitive behaviours may be associated with other 
phenotypic characteristics such as sensory processing abnormalities (Janes et al. 2014; Riby et al. 2013); it is also sugggested that they may serve to reduce anxious arousal (Riby et al. 2013; Rodgers et al. 2012).

The main aim of this study, therefore, is to describe the profile of repetitive behaviour in adults with WS and to compare this with two other genetic conditions: Prader-Willi syndrome [PWS] and Down syndrome [DS]. These were chosen as comparators since both have well-established repetitive behaviour profiles and the IQ range (mild to moderate ID) is similar to that in WS (Dykens and Rosner 1999; Martens et al., 2008; Whittington et al. 2004).

\section{Methods}

Families were contacted through the Williams Syndrome Foundation, the Prader-Willi Syndrome Association and the Downs Syndrome Association. Parents/carers received a cover letter, information sheets, consent forms and a questionnaire pack. Parents/carers were asked to assess whether the person they care for had the capacity to consent for themselves, and were provided with information to assist their decision. Several different options of consent forms were given as appropriate, with parents/carers acting as consultees to advise on the person's wishes if the person was unable to provide consent. Participants with DS and PWS were recruited as part of a large study investigating behavioural characteristics in neurodevelopmental disorders (Moss et al. 2009); individuals with WS were recruited as part of a long-term follow up study (Howlin, Elison and Stinton 2010). The majority of individuals with PWS and DS had a confirmed diagnosis from a paediatrician (PWS: 50.5\%, DS: 80.8\%) or geneticist (PWS: 24.3\%, DS: 5.1\%). In the WS sample, 76.7\% of individuals had a diagnosis confirmed by FISH testing; the remaining participants were diagnosed based on clinical features. 


\section{Participants}

Criteria for inclusion in the study were: age $\geq 18$ years and completion of all relevant questionnaire items. In the WS group, 96 primary caregivers responded to the request to participate; 90 individuals met inclusion criteria. Mean age was 33.1 years, $(S D=8.0$; range 1954); 40 (44\%) were male. In the PWS group, 303 families returned the questionnaires; 103 met inclusion criteria. Mean age was 27.9 years $(S D=8.1$; range 18-51); 49 (48\%) were male. In the DS group, 144 families completed the questionnaires; 78 participants were eligible to take part. Mean age was 30.7 years ( $S D=7.9$; range 18-47); 35 (45\%) were male. Participants with WS and DS were significantly older than those with PWS $\left(\chi^{2}(2)=22.6, p<0.01\right)$; there were no significant gender differences. Total sample size was 271.

\section{Ability levels}

As the groups were collected as part of independent studies, cognitive information varied. In the WS group, scores on the Wechsler Abbreviated Adult Intelligence Scale - Third Edition (WASI-III; Wechsler 1999) and Vineland Adaptive Behavior Scales, Second Edition (VABS; Sparrow et al., 2005) were available (WAIS-II FSIQ = 57.1, SD = 7.0; VABS Adaptive Behavior Composite $[\mathrm{ABC}]$ score $=42.2, S D=16.7)$. In the PWS and DS groups, only data on the Wessex scales, which assess functional skills, (Kushlick et al. 1973) were available (See Supplementary Materials 1 for details). The median self-help score on the Wessex was 9 (range: 0-9) for people with PWS and 9 (range: 5-9) for people with DS (maximum score=9), indicating that most individuals were able to feed, wash and dress independently. There were no significant DS vs PWS differences for verbal ability or mobility based on the Wessex scores. 


\section{Measures}

Repetitive Behaviour Questionnaire (RBQ; Moss et al. 2009). The RBQ is a 19-item informant scale designed for individuals with ID. It comprises of five subscales: insistence on sameness, compulsive behaviours, stereotyped behaviours, repetitive speech and restricted preferences (see Supplementary Materials 2, Table 1 for subscale reliability). Items are rated based on the previous month, as 'never' (0); 'once a month’ (1); 'once a week' (2); 'once a day (3); '> once a day' (4). Behaviours scoring $\geq 3$ are deemed to be of clinical significance on this measure. Test-retest reliability and inter-rater reliability at item-level for the RBQ are good, scoring within the ranges of .61-.93, and .46-.80 respectively (Moss et al. 2009).

\section{Data analysis}

Data were tested for normality using Shapiro Wilks tests. As RBQ data were not normally distributed, non-parametric tests (Kruskal Wallis, Mann-Whitney U, Chi-Square) were used to assess group differences in full scale, subscale and item scores (for clinical cut-off scores, see Supplementary Materials 3, Table 2). In the WS group, Spearman's rho correlations were used to explore correlations between the RBQ and chronological age, the WAIS-III FSIQ and VABS ABC (correlation matrix available in Supplementary Materials 4, Table 3). A p-value of .05 was used for the subscale level analyses and a conservative $p$-value of .005 was used for the item level analyses.

\section{Results}

\section{Total score, sub-scale level and item level analyses}

Median scores and post hoc analyses for the total score and subscales are presented in Table 1 (Mean scores and standard deviations are available in Supplementary Materials 5, Table 4). Individuals with WS scored significantly higher than those with DS for stereotyped behaviours; 
People with PWS scored significantly higher than those with DS for repetitive use of language. The groups did not differ on the remaining subscales or total RBQ score. Figure 1 displays the profiles of item level repetitive behaviour scores for the three groups (cf Moss et al., 2009). Overall, the profiles of repetitive behaviour were similar across groups. People with WS and PWS displayed more hoarding behaviours and less tidying behaviours than people with DS. People with WS also showed elevated levels of body stereotypy compared to those with PWS or DS, while people with PWS exhibited higher levels of repetitive questioning than those with DS. There were no significant differences between the number of repetitive behaviours shown by individuals in each group: median (range) = PWS, 2 (0-15); DS, 1 (0-17); WS, 3 (0-12).

(Insert Table 1 here)

(Insert figure 1 here)

\section{Associations with adaptive behaviour}

In the WS group, higher IQ was negatively associated with repetitive questions (rho $=-.40, p$ $<$.001). A higher VABS ABC score was negatively associated with repetitive questions (rho $=-.36, p=.001)$, adherence to routine (rho $=-.39, \mathrm{p}<.001)$ and echolalia $($ rho $=-.39, p<.001)$. The association between VABS ABC score and restricted conversation approached significance (rho $=-.29, p=.007$ ). There was no significant association between any RBQ variable and age ( $p>.005$; supplementary materials 4$)$.

\section{Discussion}

This study is the first to conduct a fine-grained analysis of profiles of repetitive behaviour amongst adults with WS. Overall, stereotyped behaviours were reported more frequently for 
WS participants than those with DS and PWS. This is consistent with previous reports of high levels of stereotypy in people with WS (Davies et al. 1998; Green et al., 2012). When comparing the groups at item-level, there were few differences, with only three behaviours body stereotypy, tidying and hoarding - differing between individuals with WS and those with PWS and/or DS.

Although hoarding is considered a prominent characteristic amongst people with PWS (Dykens et al. 1996), it has seldom been associated with WS. This finding highlights the benefits of examining repetitive behaviour at the item level and indicates that there may be behavioural difficulties that have not yet been fully acknowledged or identified in WS. Thus, recognition of increased levels of hoarding in these syndrome groups may enhance our understanding of the behaviour and its potential underlying mechanisms. For example, in anxiety research more generally, several studies have noted the association between hoarding and intolerance for uncertainty [i.e. a cognitive bias whereby individuals interpret ambiguous or uncertain situations negatively] (Dugas et al. 2004; Oglesby et al. 2013; Wheaton et al. 2016). Intolerance for uncertainty and repetitive behaviours have also been associated with anxiety in individuals with WS and with autism (Joyce et al., 2016; Rodgers et al. 2012). Since anxiety is elevated in people with PWS and WS (Richdale et al. 1999; Royston et al. 2016) the association between intolerance for uncertainty, hoarding, and other repetitive behaviours in these conditions requires further investigation.

In the WS sample, higher levels of IQ and adaptive ability were negatively associated with repetitive speech and adherence to routines. Better understanding of the relationship between ability level and repetitive behaviours is also needed to identify factors that are important in the development and maintenance of these difficulties. However, there were no other 
significant associations with ability level or age suggesting that most behaviours may be independent of developmental delay in this group.

In general, there were few significant differences between the groups and this overlap in behavioural characteristics may have implications for future research. For example in PWS, preference for predictability has been associated with certain executive function difficulties, such as task switching deficits (Woodcock et al. 2009). Thus, evaluating aspects of executive functioning in conditions such as WS may prove helpful in identifying possible causes of repetitive behaviours. A focus on specific domains of executive functioning, such as switching, inhibition and working memory/updating (Miyake \& Friedman, 2012), may also identify whether specific executive function deficits give rise to specific repetitive behaviours. For instance, in ASD, poor inhibitory control has been implicated in the presentation of compulsions (Mosconi et al. 2009).

The study has a number of limitations. Firstly, as datasets were derived from existing studies we lacked data to match participants on degree of ID or adaptive behaviour. However despite potential IQ differences, there were few differences between the groups for the majority of repetitive behaviours. Moreover, as the study focuses on adults, research in this area needs to be extended to children, to investigate developmental trajectories and changes with age.

There may also be some additional forms of repetitive behaviours that are specific to individuals with WS and which are not included in the RBQ (Moss et al. 2009). Moreover, as the RBQ only measures frequency, a tool that includes ratings of severity and impairment may strengthen the clinical utility of findings. Despite this, the RBQ is widely used in neurodevelopmental disorder research and has proven value in describing fine-grained repetitive behaviour profiles and identifying between group differences (Moss et al. 2009). A 
further potential bias is the reliance on informant reports. To provide further insight into the repetitive behaviour profile in WS, further research should consider more direct observation and, where possible, inclusion of self-report data.

Lastly, some participants did not have a confirmed diagnosis through genetic testing, as many individuals were diagnosed prior to the development of the FISH test. The study also did not account for the impact of medication use on the frequencies and topographies of repetitive behaviours. Further studies should address and improve on these limitations.

In conclusion, this study provides a detailed description of the phenomenology of repetitive behaviours in a sample of adults with WS. Overall there were few significant differences in repetitive behaviour profiles between individuals with WS and those with Prader-Willi or Down syndromes. The findings suggest the importance of further research to identify potentially shared cognitive underpinnings, and ways of ameliorating or preventing these problems that can work across syndrome groups. Finally, the study supports the utility of a fine-grained approach to investigate repetitive behaviours in genetic syndromes. 


\section{Compliance with ethical standards}

Funding: This study was funded by Cerebra, the Cornelia de Lange Foundation, Foundation LeJeune and the Williams Syndrome Foundation.

Disclosure of potential conflicts of interest: The authors have no conflict of interest to declare.

Research involving Human Participants and/or Animals: All procedures performed in studies involving human participants were in accordance with the ethical standards of the institutional and/or national research committee and with the 1964 Helsinki declaration and its later amendments or comparable ethical standards. This article does not contain any studies with animals performed by any of the authors.

Informed consent: Informed consent was obtained from individuals who had the capacity to provide consent themselves. For those who did not have capacity to provide their own consent, parents or carers were able to act as consultees. Consultees were asked to advise on what the wishes of the individual would be if they were able to consent from themselves. 


\section{References}

Davies, M., Udwin, O., \& Howlin, P. (1998). Adults with Williams syndrome - Preliminary study of social, emotional and behavioural difficulties. Br J Psychiatry, 172, 273-276, doi:10.1192/bjp.172.3.273.

Dugas, M. J., Schwartz, A., \& Francis, K. (2004). Brief report: Intolerance of uncertainty, worry, and depression. Cognitive Therapy and Research, 28(6), 835-842.

Dykens, E. M., Leckman, J. F., \& Cassidy, S. B. (1996). Obsessions and compulsions in Prader-Willi syndrome. Journal of Child Psychology and Psychiatry, 37(8), 9951002.

Dykens, E. M., \& Rosner, B. A. (1999). Refining behavioral phenotypes: Personalitymotivation in Williams and Prader-Willi syndromes. American Journal on Mental Retardation, 104(2), 158-169.

Green, T., Avda, S., Dotan, I., Zarchi, O., Basel-Vanagaite, L., Zalsman, G., ... \& Gothelf, D. (2012). Phenotypic psychiatric characterization of children with Williams syndrome and response of those with ADHD to methylphenidate treatment. American Journal of Medical Genetics Part B: Neuropsychiatric Genetics, 159(1), 13-20.

Howlin, P., Elison, S., \& Stinton, C. (2010). Cognitive, linguistic and adaptive functioning in Williams syndrome: Trajectories from early to middle adulthood. Journal of Applied Research in Intellectual Disabilities, 23, 322 - 336.

Janes, E., Riby, D. M., \& Rodgers, J. (2014). Exploring the prevalence and phenomenology of repetitive behaviours and abnormal sensory processing in children with Williams Syndrome. Journal of Intellectual Disability Research, 58(8), 746-757, doi:10.1111/jir.12086.

Joyce, C., Honey, E., Leekam, S. R., Barrett, S. L., \& Rodgers, J. (2017). Anxiety, Intolerance of Uncertainty and Restricted and Repetitive Behaviour: Insights Directly from Young People with ASD. Journal of Autism and Developmental Disorders, 114.

Kushlick, A., Blunden, R., \& Cox, G. (1973). A method of rating behaviour characteristics for use in large scale surveys of mental handicap. Psychological Medicine, 3(4), 466478.

Lincoln, A. J., Searcy, Y. M., Jones, W., \& Lord, C. (2007). Social interaction behaviors discriminate young children with autism and Williams syndrome. Journal of the American Academy of Child \& Adolescent Psychiatry, 46(3), 323-331.

Martens, M. A., Wilson, S. J., \& Reutens, D. C. (2008). Research Review: Williams syndrome: a critical review of the cognitive, behavioral, and neuroanatomical phenotype. Journal of Child Psychology and Psychiatry, 49(6), 576-608.

Miyake, A., \& Friedman, N. P. (2012). The nature and organization of individual differences in executive functions four general conclusions. Current directions in psychological science, 21(1), 8-14.

Mosconi, M., Kay, M., D'Cruz, A.-M., Seidenfeld, A., Guter, S., Stanford, L., et al. (2009). Impaired inhibitory control is associated with higher-order repetitive behaviors in autism spectrum disorders. Psychological Medicine, 39(09), 1559-1566.

Moss, J., Oliver, C., Arron, K., Burbidge, C., \& Berg, K. (2009). The prevalence and phenomenology of repetitive behavior in genetic syndromes. Journal of Autism and Developmental Disorders, 39(4), 572-588.

Oglesby, M. E., Medley, A. N., Norr, A. M., Capron, D. W., Korte, K. J., \& Schmidt, N. B. (2013). Intolerance of uncertainty as a vulnerability factor for hoarding behaviors. Journal of Affective Disorders, 145(2), 227-231. 
Pober, B. R. (2010). Medical progress: Williams-Beuren syndrome. [Review]. New England Journal of Medicine, 362(3), 239-252.

Riby, D. M., Janes, E., \& Rodgers, J. (2013). Brief Report: Exploring the Relationship Between Sensory Processing and Repetitive Behaviours in Williams Syndrome. Journal of Autism and Developmental Disorders, 43(2), 478-482, doi:10.1007/s10803-012-1557-1.

Richdale, A., Cotton, S., \& Hibbit, K. (1999). Sleep and behaviour disturbance in PraderWilli syndrome: a questionnaire study. Journal of Intellectual Disability Research, 43(5), 380-392.

Rodgers, J., Riby, D. M., Janes, E., Connolly, B., \& McConachie, H. (2012). Anxiety and Repetitive Behaviours in Autism Spectrum Disorders and Williams Syndrome : A Cross-Syndrome Comparison. Journal of Autism and Developmental Disorders, 42, Numb 2, 175-180.

Royston, R., Howlin, P., Waite, J., \& Oliver, C. (2016). Anxiety Disorders in Williams Syndrome Contrasted with Intellectual Disability and the General Population: A Systematic Review and Meta-Analysis. Journal of Autism and Developmental Disorders, 1-13.

Sparrow, S. S., Balla, D. A., \& Cicchetti, D. V. (2005). Vineland II: vineland adaptive behavior scales: American Guidance Service.

Strømme, P., Bjømstad, P. G., \& Ramstad, K. (2002). Prevalence estimation of Williams syndrome. Journal of child neurology, 17(4), 269-271.

Turner, M. (1997). Towards an executive dysfunction account of repetitive behaviour in autism.

Waite, J., Moss, J., Beck, S. R., Richards, C., Nelson, L., Arron, K., et al. (2015). Repetitive Behavior in Rubinstein-Taybi Syndrome: Parallels with autism spectrum phenomenology. Journal of Autism and Developmental Disorders, 45(5), 1238-1253.

Wechsler, D. (1999). Manual for the Wechsler abbreviated intelligence scale (WASI). San Antonio, TX: The Psychological Corporation.

Wheaton, M. G., Abramowitz, J. S., Jacoby, R. J., Zwerling, J., \& Rodriguez, C. I. (2016). An investigation of the role of intolerance of uncertainty in hoarding symptoms. Journal of Affective Disorders, 193, 208-214.

Whittington, J., Holland, A., Webb, T., Butler, J., Clarke, D., \& Boer, H. (2004). Cognitive abilities and genotype in a population-based sample of people with Prader-Willi syndrome. Journal of Intellectual Disability Research, 48(2), 172-187.

Woodcock, K. A., Oliver, C., \& Humphreys, G. W. (2009). Task-switching deficits and repetitive behaviour in genetic neurodevelopmental disorders: data from children with Prader-Willi syndrome chromosome 15 q11-q13 deletion and boys with Fragile X syndrome. Cognitive Neuropsychology, 26(2), 172-194. 
Table 1. Median scores, ranges, statistical analyses and post hoc analyses for the total scores and five subscales of the Repetitive Behaviour Questionnaire for Williams syndrome (WS), Prader-Willi syndrome (PWS) and Downs syndrome (DS)

\begin{tabular}{|c|c|c|c|c|c|c|}
\hline & \multicolumn{3}{|l|}{ Group } & \multirow{2}{*}{$x^{2}$} & \multirow[t]{2}{*}{$p$ value } & \multirow[t]{2}{*}{ Post hoc analyses } \\
\hline & $\begin{array}{l}\text { WS } \\
(n=90) \\
\text { Median (range) }\end{array}$ & $\begin{array}{l}\text { PWS } \\
(n=103) \\
\text { Median (range) }\end{array}$ & $\begin{array}{l}\text { DS } \\
(n=78) \\
\text { Median (range) }\end{array}$ & & & \\
\hline Stereotyped behaviour & $2.0(0.0-11.0)$ & $1.0(0.0-11.0)$ & $0.0(0.0-12.0)$ & 10.41 & .005 & WS $>$ DS \\
\hline Compulsive behaviour & $2.0(0.0-24.0)$ & $2.0(0.0-21.0)$ & $1.5(0.0-29.0)$ & 0.26 & .880 & \\
\hline Restricted preferences & $2.0(0.0-11.0)$ & $2.0(0.0-12.0)$ & $2.0(0.0-12.0)$ & 3.29 & .193 & \\
\hline Insistence on sameness & $1.0(0.0-8.0)$ & $3.0(0.0-8.0)$ & $2.0(0.0-8.0)$ & 5.71 & .057 & \\
\hline Repetitive use of language & $1.0(0.0-12.0)$ & $2.0(0.0-12.0)$ & $0.0(0.0-12.0)$ & 12.18 & .002 & PWS $>$ DS \\
\hline Total score & $11.8(0.0-49.0)$ & $12.0(0.0-60.0)$ & $8.0(0.0-66.0)$ & 3.64 & .162 & \\
\hline
\end{tabular}


Figure 1. Mean item level scores depicting the frequency of each type of repetitive behaviour for each syndrome. Shaded areas represent each subscale. A '+' indicates a group scored significantly higher than one other group, a '- 'indicates a significantly lower score than one other group. (See Supplementary Materials 6 for further explanation)
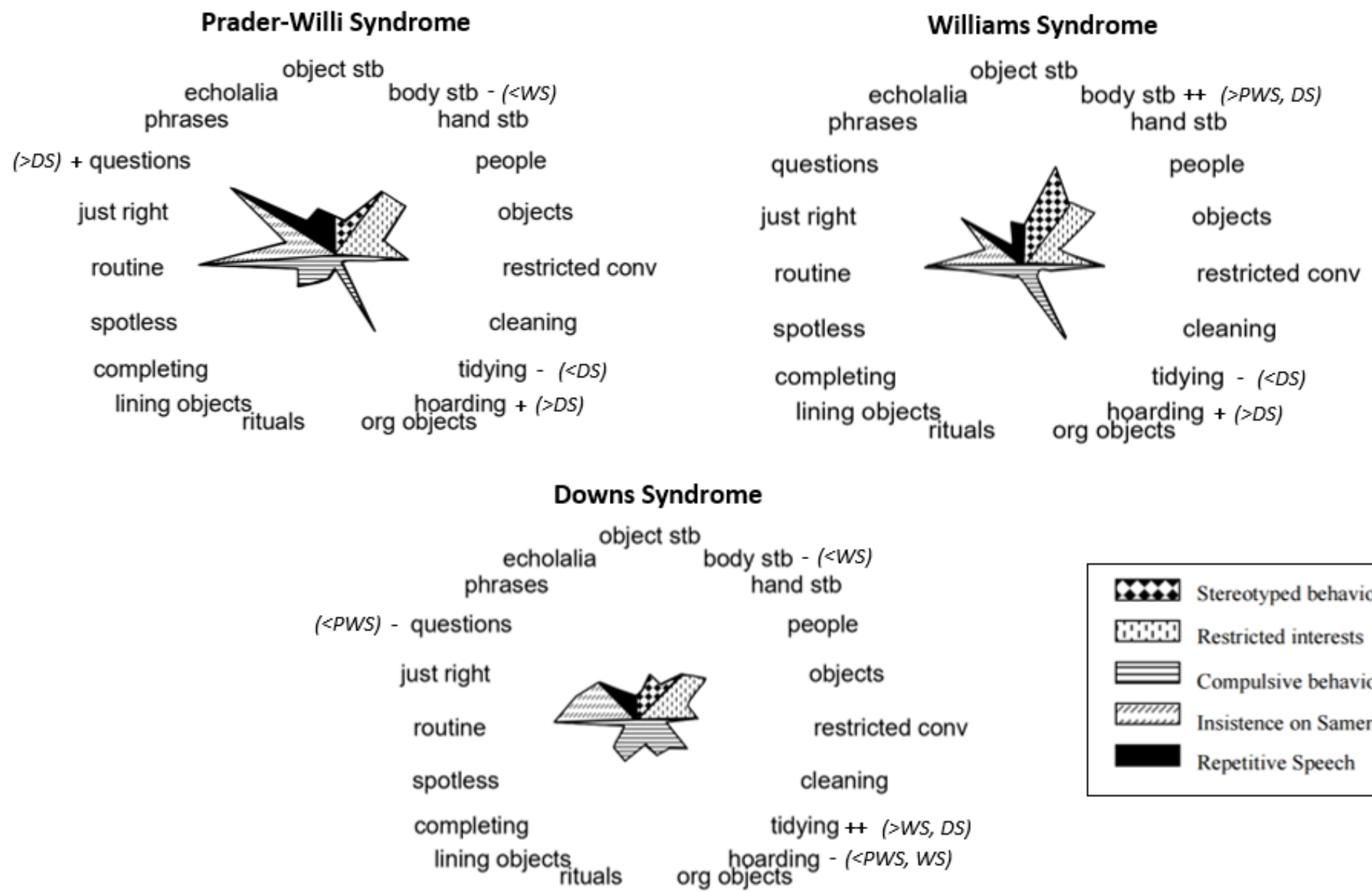